\title{
The Central Projection of Cephalic Mechanosensory Axons in the Haematophagous Bug Triatoma infestans
}

\author{
Teresita C Insausti ${ }^{+}$, Claudio R Lazzari \\ Departamento de Biología, Facultad de Ciencias Exactas y Naturales, Universidad de Buenos Aires, \\ Ciudad Universitaria, (1428) Buenos Aires, Argentina
}

The projections of mechanosensory hairs located on the dorsal and lateral head of the adult haematophagous bug Triatoma infestans were analyzed by means of cobalt filling. Axons run into the anterior and posterior tegumentary nerve and project through the brain to the ventral nerve cord. The fibres are small in diameter and run as a fascicle. Some branches run into suboesophageal and prothoracic centres; others run as far as to the mesothoracic ganglion. These sensory projections resemble that of wind-sensitive head hairs of the locust. The functional role of this sensory system in this species is discussed.

Key words: Triatoma - mechanoreceptors - Chagas disease vectors - neuroanatomy

Since the classical work of Wigglesworth and Gillett (1934) on the role of antennae during the orientation of Rhodnius prolixus, the sensory systems of Chagas disease vectors have been the subject of several studies. This work and the subsequent ones by diverse authors have allowed us to comprehend better how these insects acquire and respond to information from the environment in order to locate resources, such as food, shelters and mates. Comparative analysis with other insects reveals many unusual features in Triatominae, such as sensitivity to infrared radiation (Lazzari \& Núñez 1989), their singular anemotactic response to odours (Taneja \& Guerin 1995) or two visual systems mediating phototaxis (Lazzari et al. 1998). Of special interest due to its role in dispersal are those sensory systems involved in guiding flight and in the selection of a shelter. Both fight control and thigmotaxis involve mechanoreceptive inputs from different parts of the body.

The head of the bug Triatoma infestans is elongated and exhibits a tubular form. Compound eyes are positioned laterally. Two well-developed ocelli are located behind them, looking dorsolaterally and frontally. The whole cephalic capsule is covered

This work received financial support from the Universidad de Buenos Aires, Conicet/Argentina, and the WHO/UNDP/World Bank Special Programme for Research and Training in Tropical Diseases.

${ }^{+}$Corresponding author. Fax: +54-11-4576.3384 / 4544. 7893. E-mail: lazzari@bg.fcen.uba.ar

Received 20 September 1999

Accepted 10 January 2000 by thick sensilla chaetica, to which a mechanosensory function has been ascribed (Wigglesworth \& Gillett 1934, Bernard 1974, Lazzari 1983).

Two bilateral tegumentary nerves innervate the head integument of $T$. infestans, which gather the axons of cephalic mechanoreceptors. The anterior tegumentary nerve runs into the deutocerebrum, close to the origin of the antennal nerve, while the posterior tegumentary nerve runs into the posterior protocerebrum, close to the corpora cardiaca nerve I (Insausti 1994).

The projection of head hairs of $T$. infestans is interesting for comparative analysis for several reasons. First, the head hairs do not differ in their morphology from mechanoreceptors elsewhere on the body, i.e., heavy sensilla chaetica. Second, in other insects, head hairs are long and trichoid, with a basal plate situated on a thin flexible articular membrane (Weis-Fogh 1949), facilitating stimulation by air-currents. Finally, T. infestans, although able to fly (Lehane \& Schofield 1976, Schofield et al. 1992), are mainly walking insects, in contrast to other insects where these hairs exhibit a high degree of specialisation, such as for example, locusts.

In this study, the projections of the head hairs sensory axons within the central nervous system in the bug T. infestans are described on the basis of cobalt fillings. This method has been successfully employed in the past to reveal the ocellar pathways in this species (Insausti \& Lazzari 1996) and will be the first report on this subject for Hemiptera.

\section{MATERIALS AND METHODS}

Adults of T. infestans were reared in the laboratory at $28^{\circ} \mathrm{C}$ and fed on citrated sheep blood with 
the aid of an artificial feeder (Núñez \& Lazzari 1990).

For light microscopy, heads were fixed in a mixture of $2.5 \%$ glutaraldehyde and $2 \%$ paraformaldehyde in phosphate buffer $(\mathrm{pH} 7.3)$ and postfixed with $1 \%$ osmium tetroxide. After dehydration, the heads were embedded in Durcupan, cut at a thickness of $5 \mu \mathrm{m}$ and stained with methylene blue.

Scanning electron microscopy was done on fresh or fixed heads, using an Electroscan environmental scanning electron microscope, which does not require coating the specimen with metal.

Fifty insects were used for $\mathrm{CoCl}_{2}$ filling of the sensory axons of the dorsal head hairs. The insects were immobilised with plasticene on a glass plate, dorsal side up, with the head fixed with dental wax. Some mechanosensory hears (see below) were cut off with a razor blade and a drop of $2.5 \%$ cobalt chloride in distilled water was placed on the lesion, which was then covered with vaseline. The preparations were left for $1-3 \mathrm{~h}$ at $21^{\circ} \mathrm{C}$ for cobalt uptake. Thereafter, the animals were glued on a wooden plate with dental wax, ventral side up. The brain and ganglia were exposed and immersed for $5 \mathrm{~min}$ in a solution of ammonium sulphide (five drops of saturated ammonium sulphide added to 5 $\mathrm{ml}$ Ringer). The dissected nervous system was fixed for $2 \mathrm{~h}$ in glacial acetic acid/ethanol/formalin fixative (Lillie 1965) and silver intensified according to Bacon and Altman (1977). The cobaltlabelled tissue was either cleared with methyl salicylate for whole mount viewing or embedded in Durcupan or cut horizontally at 30-35 $\mu \mathrm{m}$. Photographs and reconstruction drawings were made from whole mounts and sections.

Cobalt applications were done at four different areas of the dorsal head capsule, i.e., (1) anterior to the compound eyes, (2) between the compound eyes, (3) around the ocelli and (4) posterior to the ocelli (Fig. 1). The last area served as a control for possible diffusion through the internal integument, given that it lacks hairs. All hairs in a given area were cut off and, in most cases, bilateral applications were performed.

Nervous areas were named according to Strausfeld (1976) and Insausti (1994).

\section{RESULTS}

Figs 1 and 2 show the distribution and general structure of the sensilla chaetica on the cephalic capsule of $T$. infestans.

The anterior and posterior tegumentary nerves collect the axons of neurones associated with hairs located in the cephalic capsule.

Axons from areas 1 and 2 (see Fig. 1) join in the anterior tegumentary nerve, proximal to the antennal nerve (Figs 3, 4, 5A). The fibres bundle runs along the brain, towards the suboesophageal ganglion. There some fibres terminate in the mandibular and maxillary neuropils (Fig 3, 4, 5B, C). Other fibres continue to the prothoracic ganglion. Some run to the midline, where their projections superimpose with those of the contralateral fascicle (Figs 3, 6A, B). From this point, axons further descend to the posterior ganglion where they send branches to the mesothoracic neuropil, where they converge in the midline together with contralateral projections (Figs 3, 6A, B).

Axons from area 3 run through the posterior tegumentary nerve into the brain and join the fibre bundles of the anterior tegumentary nerve in the suboesophageal ganglion, prior to branching into this nervous mass. From this point onwards, these axons exhibit the same branching pattern as the fibre bundles of the areas 1 and 2 (Figs 3, 4, 5B, C, $6 \mathrm{~A}, \mathrm{~B})$. Conversely to the axons projecting from areas 1 and 2, these axons penetrate the dorsal brain before running ventrally into the nerve cord.

\section{DISCUSSION}

Head hairs have been implicated in sensing air currents and initiating and maintaining flight of locusts (Weis-Fogh 1949, Wilson 1961). In addition, Neese (1965) ascribed a function related to flight control to interommatidial hairs of bees. Similar groups of hairs are also present on the head of non-flying insect species, e.g., the cricket Gryllus campestris, thus suggesting that these mechanosensors could be involved in other behaviours such as grooming. A comparative analysis of the central projection of these mechanoreceptors between different species reveals similarities. The axons enter the brain through tegumentary nerves (dorsal and ventral nerves in locusts, lateral-dorsal and medial-dorsal nerves in honeybees and dorsal nerve in crickets) and arborize in the median ventral tract of several neuromeres of the ventral cord, i.e., suboesophageal and pro- and mesothoracic ganglia (Mobbs 1985).

The central projection of cephalic mechanoreceptors of $T$. infestans is similar to that of head hairs of other insects. This is surprising because in $T$. infestans (1) the sensilla differ considerably in their morphology from wind receptors; (2) these hairs are distributed over the whole body; (3) although capable of flying, flight is restricted to certain phases and conditions of the life cycle, and (4) grooming of the antennae and legs but not groom of the head has been observed (personal observation).

Wind-sensitive hairs of the locust are long thin sensilla trichoidea that are deflected by air currents. 
They project directly into the motor centres located in the suboesophageal and pro- and mesothoracic ganglia. These characteristics indicate a fast system that is involved in the control of flight manoeuvres (Weis-Fogh 1949, Tyrer et al. 1979). However, such properties are not specific for wind- sensitive hairs. Head hairs of crickets and interommatidial bristles of honeybees, as well as other head hairs of orthopterans, also project into the medial ventral tract (Mobbs 1985). Similar descending pathways are also exhibited by $T$. infestans.

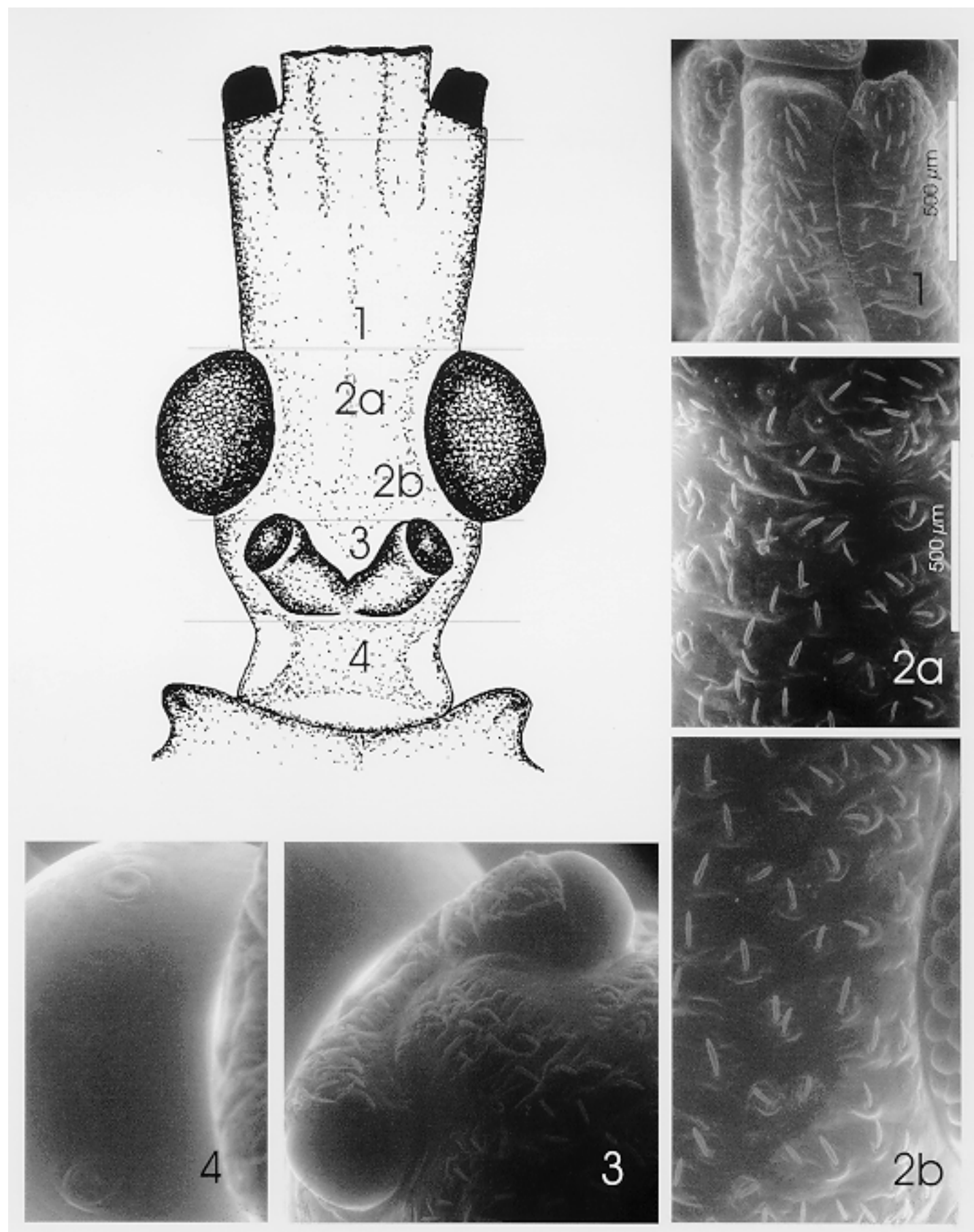

Fig. 1: schematic drawing and environmental scanning electron microscope photomicrograph of the dorsal cephalic capsule of Triatoma infestans showing the structure and distribution of the sensilla chaetica. The different areas where cobalt was applied are shown. 


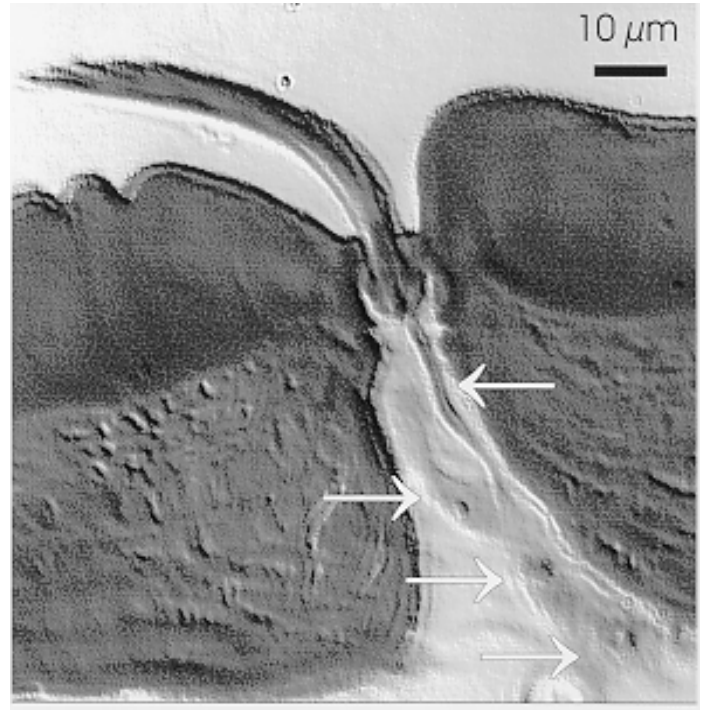

Fig. 2: section through a cephalic sensilla chaetica of Triatoma infestans showing the dendrites and associated cells (arrows).

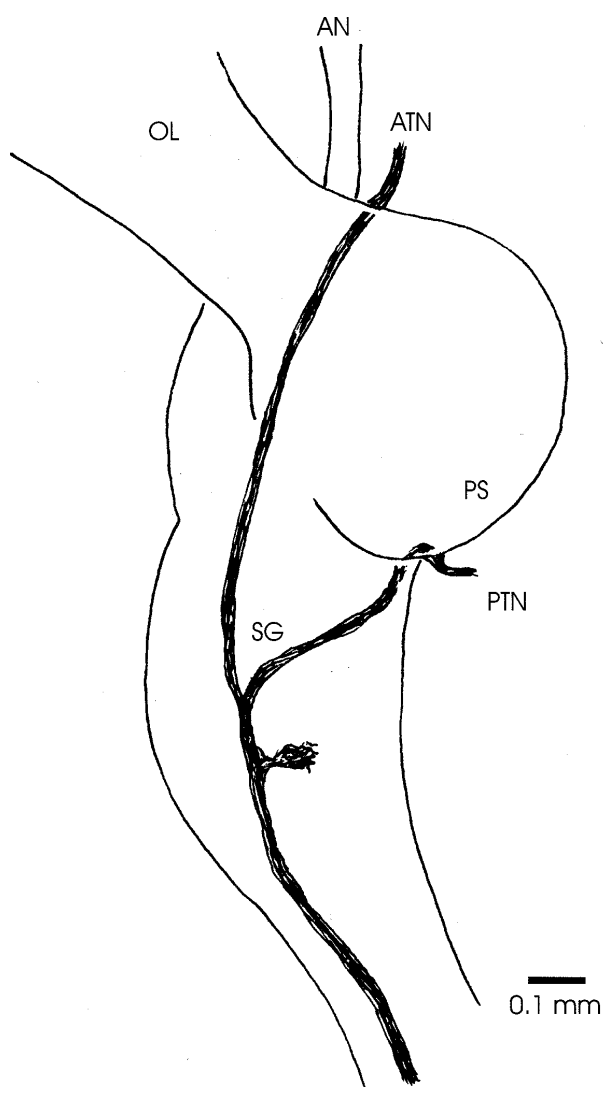

Fig. 4: tracing of cobalt-filled sensory axons of the dorsal head hairs of Triatoma infestans from whole-mount preparation. Schematic diagram of the brain, lateral viewed. AN: antennal nerve; ATN: anterior tegumentary nerve; OL: optic lobe; PS: posterior slope; PTN: posterior tegumentary nerve; SG: suboesophageal ganglion.

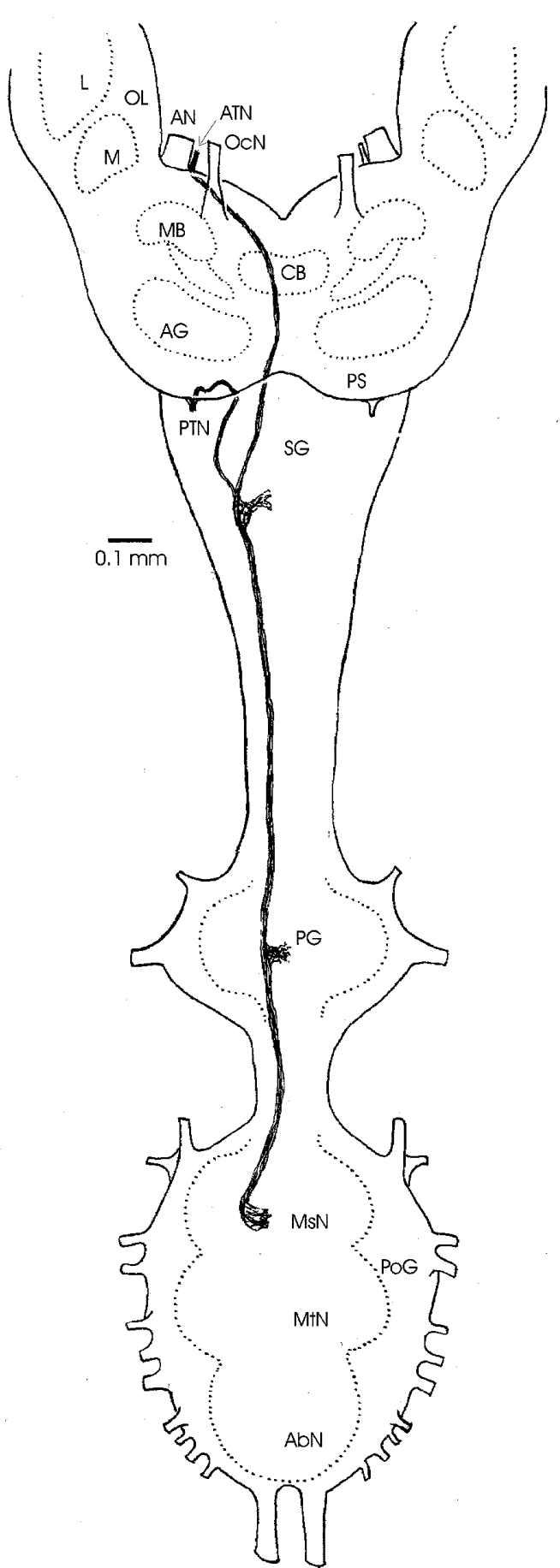

Fig. 3: tracing of cobalt-filled sensory axons of the dorsal head hairs of Triatoma infestans, as seen in whole-mount preparation. Schematic diagram of the central nervous system (dorsal view) showing some of the tracts and neuropil regions. AbN: abdominal neuropil; AG: antennal glomerulus; AN: antennal nerve; ATN: anterior tegumentary nerve; $\mathrm{CB}$ : central body; M: medulla; MB: mushroom body; L: lobula; MsN: mesothoracic neuropil; MtN: metathoracic neuropil; OcN: ocelar nerve; OL: optic lobe; PG: prothoracic ganglion; PoG: posterior ganglion; PS: posterior slope; PTN: posterior tegumentary nerve; SG: suboesophageal ganglion. 

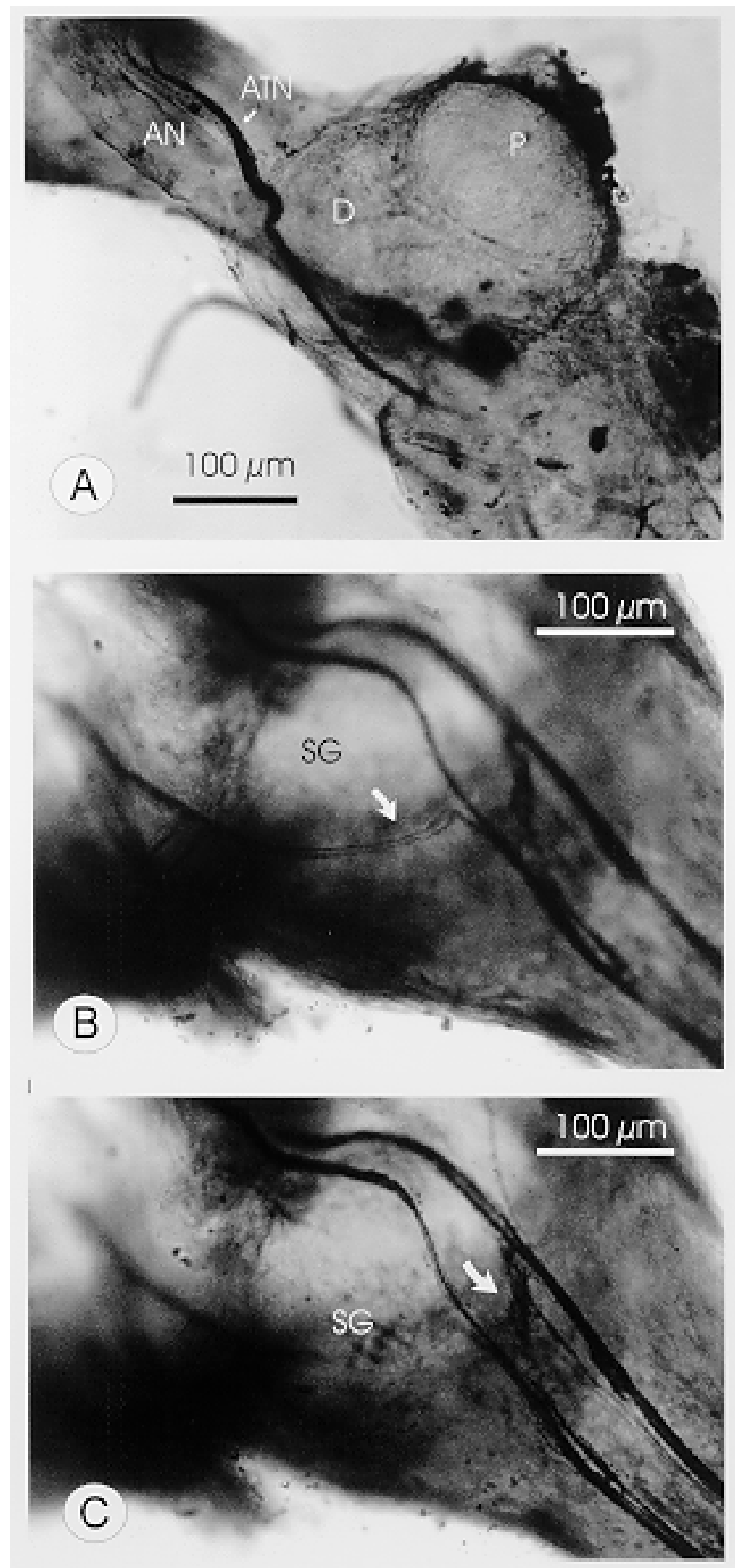

Fig. 5: micrographs of cobalt-filled sensory axons of the dorsal head hairs of Triatoma infestans as seen in whole-mount preparation. A: brain, ventral view. Stained fibres from zones 1 and 2 enter the brain through the anterior tegumentary nerve; B: ventral view of the posterior brain and the suboesophageal ganglion. Fibres coming through the posterior tegumentary nerve (arrow) join those from the anterior nerve in the suboesophageal ganglion, forming a unique fascicle; C: same preparation as B, but focused at a different level. Bilateral fibres coming through the right and left anterior tegumentary nerves run parallel to each other and arborize, together with those from the posterior tegumentary nerve, in the suboesophageal ganglion (arrow). Abbreviations as in Fig. 3. 

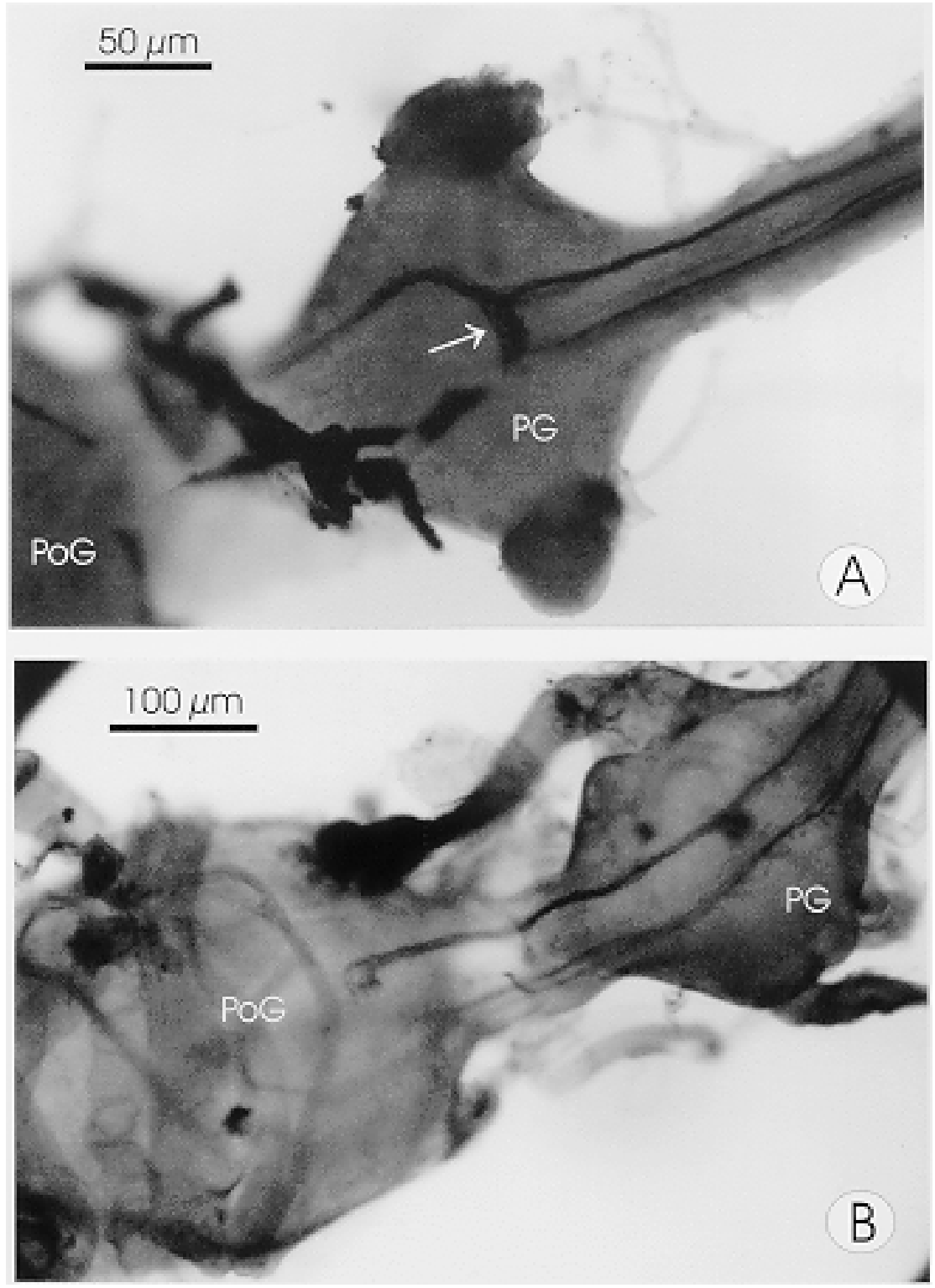

Fig. 6: micrographs of cobalt-filled sensory axons of the dorsal head hairs of Triatoma infestans, as seen in whole-mount preparations. A: dorsal view of the prothoracic ganglion after bilateral injection of hairs, showing the arborization of the fibres (arrow); B: dorsal view of the prothoracic and posterior ganglia, after unilateral injection of hairs from the zone 3. Axons arborize in the proand mesothoracic neuropil. A second fascicle can be distinguished right to the former, which correspond to first order ocellar interneurones, secondarily stained in the preparation. Abbreviations as in Fig. 3.

Comparative analysis of our present results in T. infestans and observations by other authors in other insect species suggests that direct descending projections of cephalic mechanoreceptors may not have evolved to serve a function that requires rapid processing of sensory information. An alternative hypothesis is that cephalic mechanoreceptors would be a part of a general mechanical sense, distributed all over the integument and with integrative centres located along the ventral cord. T. infestans would represent a primitive undifferentiated state, where all mechanoreceptors are morphologically identical and are gathered together in given nervous pathways. Wind receptors of the locust, interommatidial and head hairs of the honeybee and head hairs of crickets would represent morphological and functional adaptations to certain tasks such as flight, grooming, etc. 
In agreement with this hypothesis, cobalt fills of other nerves, e.g., antennal nerves in T. infestans (Insausti unpubl.), bees and locusts, mandibular nerve of the locust and neck hairs of bees (Mobbs 1985), project into the median ventral tract and overlap with other sensory projections in ganglionic motor areas. As stated by Mobbs (1985), these results suggest that the median ventral tract is an area for the association of somatosensory inputs, and indicate the extent of the direct connection between different neuromeres by populations of primary afferents.

Finally, in considering the functional role of these mechanoreceptors in $T$. infestans, the bugs spend daylight hours hidden in wall crevices and places that offer a close contact with the substrate and conspecifics. This behaviour is mediated by negative phototaxis (Reisenman et al. 1998, Lazzari et al. 1998), by assembling signals (Schofield \& Patterson 1977, Lorenzo \& Lazzari 1996, Lorenzo Figueiras \& Lazzari 1998) and by thigmotaxis. The last response is mediated by mechanoreceptive inputs coming from the whole body surface, is able to induce certain physiological changes in the bugs, such as a decrease in the heart rate (Chiang et al. 1992). Thus, head hairs could play a role in maintaining thigmotaxis and triggering physiological responses associated with this behaviour. This hypothesis receives support from the preliminary analysis of the central projection of sensilla chaetica located in different abdominal segments, as revealed by cobalt fillings (Insausti, unpubl.). Sensory axons from these mechanoreceptors branch in the same neuropil areas (meso- and metathoracic centres) as do those originating from cephalic hairs.

\section{ACKNOWLEDGMENTS}

To J Zeil for critical reading of the manuscript and for introducing to us the technique of cobalt staining. To the staff members of our laboratory for helpful discussions and improvement of the manuscript.

\section{REFERENCE}

Bacon JP, Altman JS 1977. A silver intensification method for cobalt-filled neurons in wholemount preparations. Brain Res 138: 359-363.

Bernard J 1974. Etude Électrophysiologique de Récepteurs Impliqués dans l'Orientation vers l'Hote et dans l'Acte Hématophage chez un Hémiptère: Triatoma infestans, Thesis, Université Rennes, 285 pp.

Chiang RG, Chiang JA, Davey KG 1992. A sensory input inhibiting heart rate in an insect, Rhodnius prolixus. Experientia 48: 1122-1125.

Insausti TC 1994. Nervous system of Triatoma infestans. J Morphol 221: 343-359.

Insausti TC, Lazzari CR 1996. Central projections of first-order interneurons in bug Triatoma infestans
(Heteroptera: Reduviidae). J Morphol 229: 161-169. Lazzari CR 1983. Estudio de los Receptores Sensoriales de las Antenas de Triatoma infestans (Klug, 1834) (Heteroptera:Reduviidae), Thesis, Licenciatura, Universidad de Buenos Aires, 81 pp.

Lazzari CR, Núñez JA 1989. The response to radiant heat and the estimation of the temperature of distant sources in Triatoma infestans. J Insect Physiol 35: 525-529.

Lazzari CR, Reisenman CE, Insausti TC 1998. The role of the ocelli in the phototactic response of Triatoma infestans (Heteroptera: Reduviidae). J Insect Physiol 44: 1159-1162.

Lehane MJ, Schofield CJ 1976. Preliminary report on flight by some triatomine bugs. Trans $R$ Soc Trop Med Hyg 70: 526.

Lillie RD 1965. Histopathologic Technic and Practical Histochemistry, McGraw-Hill, New York-London.

Lorenzo Figueiras AN, Lazzari CR 1998. Aggregation in the haematophagous bug Triatoma infestans: a novel assembling factor. Physiol Entomol 23: 3337.

Lorenzo MG, Lazzari CR 1996. The spatial pattern of defecation in Triatoma infestans and the role of faeces as a chemical mark of the refuge. J Insect Physiol 42: 903-907.

Mobbs PG 1985. Brain structure. In GA Kerkut \& LI Gilbert (eds), Comparative Insect Physiology, Biochemistry and Pharmacology, vol. 5: Nervous system: Structure and motor function, Pergamon Press, Oxford, p. 300-370.

Neese V 1965. Zur Funktion der Augenborsten bei der Honigbiene. Z vergl Physiol 49: 543-585.

Núñez JA, Lazzari CR 1990. Rearing of Triatoma infestans Klug (Het., Reduviidae) in the absence of a live host. I. Some factors affecting the artificial feeding. J Applied Entomol 109: 87-92.

Reisenman CE, Lazzari CR, Giurfa M 1998. Circadian control of photonegative sensitivity in the haematophagous bug Triatoma infestans. J Comp Physiol A 183: 533-541.

Schofield CJ, Patterson JW 1977. Assembly pheromone of Triatoma infestans and Rhodnius prolixus nymphs (Hemiptera: Reduviidae). J Med Entomol 13: 727 734.

Schofield CJ, Lehane MJ, McEwen PK, Catala SS, Gorla DE 1992. Dispersive flight by Triatoma infestans under natural climatic conditions in Argentina. Med Vet Ent 6: 51-56.

Strausfeld NJ 1976. Atlas of an Insect Brain, Springer Berlin, Heidelberg, New York, 214 pp.

Taneja J, Guerin P M 1995. Oriented responses of the triatomine bugs Rhodnius prolixus and Triatoma infestans to vertebrate odours on a servosphere. $J$ Comp Physiol A 176: 455-464.

Tyrer NM, Bacon, JP, Davies CA 1979. Sensory projections from the wind-sensitive head hairs of the locust Schistocerca gregaria. Cell Tiss Res 203: 79-92.

Weis-Fogh T 1949. An aerodynamic sense organ stimulating and regulating flight in locusts. Nature 164: 873-874. 
Wigglesworth VB, Gillett JD 1934. The function of the antennae in Rhodnius prolixus (Hemiptera) and the mechanism of orientation to the host. J Exp Biol 11:
120-138

Wilson DM 1961. The central nervous control of flight in locust. J Exp Biol 38: 471-490. 